\title{
Identification of emergency department patients for referral to rapid-access addiction services
}

\author{
Jessica Hann (D, MD*†; Howie Wu, BSc ${ }^{\dagger}$; Aliyah Gauri, $\mathrm{MSPH}^{*}$; Kathryn Dong, MD, MSc, FRCPC ${ }^{*}+\S$; \\ Ni Lam, MD, PhD, FRCPC ${ }^{*}$; Jeffrey A. Bakal, PhD, P.Stat"; Allison Kirkham, MD, FRCPC*†
}

\begin{abstract}
CLINICIAN'S CAPSULE
What is known about the topic?

Substance-related emergency department (ED) visits are rapidly increasing, yet many EDs do not have referral protocols for rapid-access addiction services.

What did this study ask?

This study characterized substance-related ED presentations and assessed need for a rapid-access addiction clinic for direct referral from the ED.

What did this study find?

There is a need for a rapid-access addiction clinic, given that four ED patients would have been referred per day. Why does this study matter to clinicians?

Creating a rapid-access addiction clinic could benefit an underserved patient population and directly connect patients to addiction follow-up care from the ED.
\end{abstract}

\section{ABSTRACT}

Objectives: Substance-related emergency department (ED) visits are rapidly increasing. Despite this finding, many EDs do not have access to on-site addiction services. This study characterized substance-related ED presentations and assessed the ED health care team's perceived need for an on-site rapid-access addiction clinic for direct patient referral from the ED.

Methods: This prospectively enrolled cohort study was conducted at an urban tertiary care ED from June to August 2018. Adult ED patients with problematic or high-risk substance use were enrolled by ED staff using a one-page form. The electronic and paper records from the index ED visit were reviewed. The primary outcome evaluated whether the ED health care team would have referred the patient to an on-site rapid-access addiction clinic, if one were available.

Results: We received 557 enrolment forms and 458 were included in the analysis. Median age was 35 years, and $64 \%$ of included patients were male. Alcohol was the most commonly reported substance of problematic or high-risk use $(60 \%)$. Previous ED visits within 7 days of the index visit were made by $28 \%$ of patients. The ED health care team indicated "Yes" for rapid-access addiction clinic referral from the ED for $66 \%$ of patients, with a mean of 4.3 patients referred per day during the study period.

Conclusions: At least four patients per day would have been referred to an on-site rapid-access addiction clinic from the $E D$, had one been available. This indicates a gap in care and collaborating with other sites that have successfully implemented this clinic model is an important next step.

\section{RÉSUMÉ}

Objectifs: Le nombre de consultations au service des urgences (SU) pour des troubles liés à l'utilisation de drogues connaît une augmentation rapide. Toutefois, bon nombre de SU ne disposent pas de service d'aide aux toxicomanes, sur place. L'étude visait donc à caractériser les consultations au SU motivées par des troubles liés à I'utilisation de drogues, et à évaluer la perception de l'équipe de soins au SU quant à la nécessité d'un service d'aide rapide aux toxicomanes, sur place, pour les patients provenant directement du SU.

Méthode: II s'agit d'une étude de cohortes, prospective, menée dans un SU de soins tertiaires, en milieu urbain, de juin à août 2018. Les adultes examinés au SU et connaissant des problèmes ou des risques élevés de consommation de drogues ont été inscrits à l'étude par le personnel du SU, à l'aide d'un formulaire d'une page. Les dossiers électroniques et les dossiers sur papier concernant la visite de référence au SU ont fait l'objet d'examen. Le principal critère consistait en I'évaluation du personnel du SU quant à la pertinence d'orienter des patients vers un service $d^{\prime}$ 'aide rapide aux toxicomanes, sur place, s'il y en avait eu un.

Résultats: L'équipe a reçu 557 formulaires d'inscription, et 458 patients ont été inclus dans l'étude. L'âge médian s'élevait à 35 ans, et $64 \%$ des sujets retenus étaient des hommes. D'après le

From the ${ }^{*}$ University of Alberta Department of Emergency Medicine, Edmonton, AB, Canada; ${ }^{\top}$ University of Alberta Faculty of Medicine \& Dentistry, Edmonton, AB, Canada; ${ }^{\ddagger}$ British Columbia Centre on Substance Use, Vancouver, BC, Canada; ${ }^{\S}$ Inner City Health and Wellness Program, Royal Alexandra Hospital, Edmonton, AB, Canada; and the "Provincial Research Data Services, Alberta Health Services, Edmonton, AB, Canada.

Correspondence to: Dr. Jessica Hann, 2J2.00 WC Mackenzie Health Sciences Centre, 8440112 St NW, Edmonton, Alberta, T6G 2R3, Canada; Email: jehann@ualberta.ca 
formulaire d'inscription, la drogue causant le plus de problèmes ou associée le plus souvent à une consommation risquée $(60 \%)$ était l'alcool. Par ailleurs, $28 \%$ des patients étaient déjà allés au SU, au cours des 7 jours précédant la consultation de référence. Dans $66 \%$ des cas, l'équipe de soins au SU a indiqué "Oui " quant à la nécessité de diriger des patients vers un service d'aide rapide aux toxicomanes; ainsi, 4,3 patients par jour, en moyenne, auraient été orientés vers ce type de soutien, durant la période à l'étude.
Conclusion: Au moins 4 patients par jour, provenant directement du SU, auraient été dirigés vers un service d'aide rapide aux toxicomanes, sur place, s'il y en avait eu un. Les résultats font ressortir une lacune dans la prestation des soins, et la collaboration avec des centres qui ont déjà réussi à mettre sur pied ce genre de service clinique représente une étape importante.

Keywords: Substance use, quality improvement, emergency medicine

\section{INTRODUCTION}

Emergency department (ED) visits related to substance use are rapidly increasing. ${ }^{1-4}$ Opioid-related deaths have surpassed trauma as the leading cause of death in persons age 30 to 39 in both Canada and the United States. ${ }^{3,5-7}$ National public health officials in both countries have declared this a major public health crisis. ${ }^{8}$ The $\mathrm{ED}$ is a major point of health care access for patients who use substances. ${ }^{9}$ Previous Canadian studies observed that people who use drugs use the ED at a rate 7 times that of a cohort-matched population, and that substance use contributes significantly to ED visits, hospital admissions, and duration of ED stay. ${ }^{9,10}$ This demonstrates the ideal position of the ED to identify and intervene upon patients with high-risk substance use.

There are currently many EDs that do not provide immediate access to evidence-based treatment for substance use disorders. Connecting patients with on-site addiction services after discharge is similarly challenging. ${ }^{1-14}$ Research addressing the impact of access to addiction services for ED patients is limited. However, the importance of ED patients having access to immediate on-site addiction care is demonstrated by a 2017 study of over 12,000 patients which found that $6.5 \%$ of patients who had an opioid overdose reversed by naloxone died later on the same day. ${ }^{15}$

The primary objective of this prospectively enrolled cohort study was to identify the potential need for an on-site rapid-access addiction clinic. This was done by surveying the patient's health care team about whether they would have referred the patient to this clinic following an ED visit related to substance use, had the clinic been available. We secondarily aimed to characterize this patient population and features of their ED visit. The rapid-access addiction clinic model would deliver services including assessment by an addiction medicine physician, pharmacotherapy (such as opioid agonist treatment), addiction counseling, harm reduction counseling and supplies, and social stabilization. Previous literature outlining the impact of rapid-access addiction clinics is limited, but several North American studies have demonstrated that connecting patients from hospital to specialized addiction resources leads to a reduction in repeat ED visits. ${ }^{16-21}$

\section{METHODS}

\section{Design}

Prospective patient enrolment took place over a 10-week period from June 13 to August 21, 2018. ED patients with known or suspected problematic or highrisk substance use were enrolled by any member of their health care team (including the attending ED physician, resident physician, medical student, bedside nurse, or social worker) or our research team using a one-page form. Risk of substance use was determined by the bedside judgment of the health care team member. A formal screening process for substance use was not used. ED staff were made aware of the study at regularly scheduled meetings, and information posters were displayed in the ED.

Patient consent for study enrolment was waived. The study was approved by the Health Research Ethics Board at the University of Alberta.

\section{Setting}

This study was conducted at the University of Alberta Hospital ED, a tertiary care trauma center in Edmonton, 
Alberta, Canada (an urban center with a population of approximately 1 million residents). This ED had 67,511 unique adult visits in 2018, equaling approximately 185 visits per day.

\section{Subjects}

We included all adult patients (age $\geq 18$ years) with known or suspected problematic or high-risk substance use (as determined by the bedside judgment of the ED health care team). We excluded patients who were medically unstable requiring hospital admission, patients who were directly referred to the ED for hospital admission by a consulting service, and patients in corrections custody, as these patients would not be eligible for referral to an outpatient clinic. We also excluded any patient whose substance use history was unable to be assessed (for any reason) at any point in their ED visit.

\section{Data collection}

The enrolment form collected patient demographics, substance use history, suitability for services that would be offered at a rapid-access addiction clinic, and asked the enrolling party to indicate if they believed the patient would be appropriate for referral to this clinic, if it were available. Patients were not directly asked if they would accept this referral. The index ED visit for all included patients underwent both paper and electronic chart review (by means of the Emergency Department Information System) by one reviewer. Enrolment forms were stored by the ED unit clerk for collection by our research team. Abstraction of data was done using an encrypted Excel spreadsheet that was stored on a passwordprotected computer. Only study investigators had access to this spreadsheet.

\section{Outcome measures}

The primary outcome evaluated whether the ED health care team would have referred the patient to an on-site rapid-access addiction clinic, if one were available. This was assessed using the study enrolment form, which asked the enrolling party to indicate "Yes" or "No" for patient referral from the ED. We secondarily aimed to characterize this patient population and features of their ED visit.

\section{Data analysis}

Descriptive analyses were used to summarize patient demographic and ED visit characteristics. Summary findings are presented as frequencies, percentages, medians, and interquartile ranges (IQR), as appropriate. To investigate factors that were significantly associated with patient referral to a hypothetical on-site rapidaccess addiction clinic, we conducted a series of multivariable logistic regression models, which included the following variables: patient age (years), sex (male or female), Canadian Triage Acuity Scale (CTAS) score, ED triage complaint category, substance(s) of use, date and time of ED triage and discharge, ED length of stay (hours and minutes), and repeat ED visits in the previous 7 days and 30 days. ${ }^{22}$ These variables were selected a priori, and we used the stepwise forward selection procedure to build the final model. Variables were examined for multicollinearity and leverage, and model residuals were graphically examined for fit. Additionally, we considered a multilevel model with weekday level but found no significant changes in this model. Model fit was assessed by the c-index. We report adjusted odds ratios (aORs) with corresponding 95\% confidence intervals (CIs). Data management and analysis were performed using statistical software (SAS v9.4, SAS Institute Inc., Cary, NC, USA).

\section{RESULTS}

During the study period, there were 11,450 visits to the University of Alberta Hospital ED, of which 7,833 were discharged patients. We received 557 enrollment forms and 458 were included in the analysis (Figure 1). A total of 99 forms were excluded; 80 patients did not meet inclusion criteria (50 patients were unable to have their substance use history assessed, 26 were admitted to hospital, and 4 were in corrections custody), and 9 patients had duplicate enrolment forms completed for the same ED visit, corresponding to a total of 19 forms (Figure 1). Of the 458 included forms, 65.7\% indicated "Yes" and 34.3\% indicated "No" for patient referral to a hypothetical rapid-access addiction clinic from the ED. Patients recorded as "Would not accept referral" indicates that the referring party believed the patient would decline referral if offered (but the patient was not consulted directly to assess this). Reasons listed as "Other" for not referring a patient (38 patients) included 


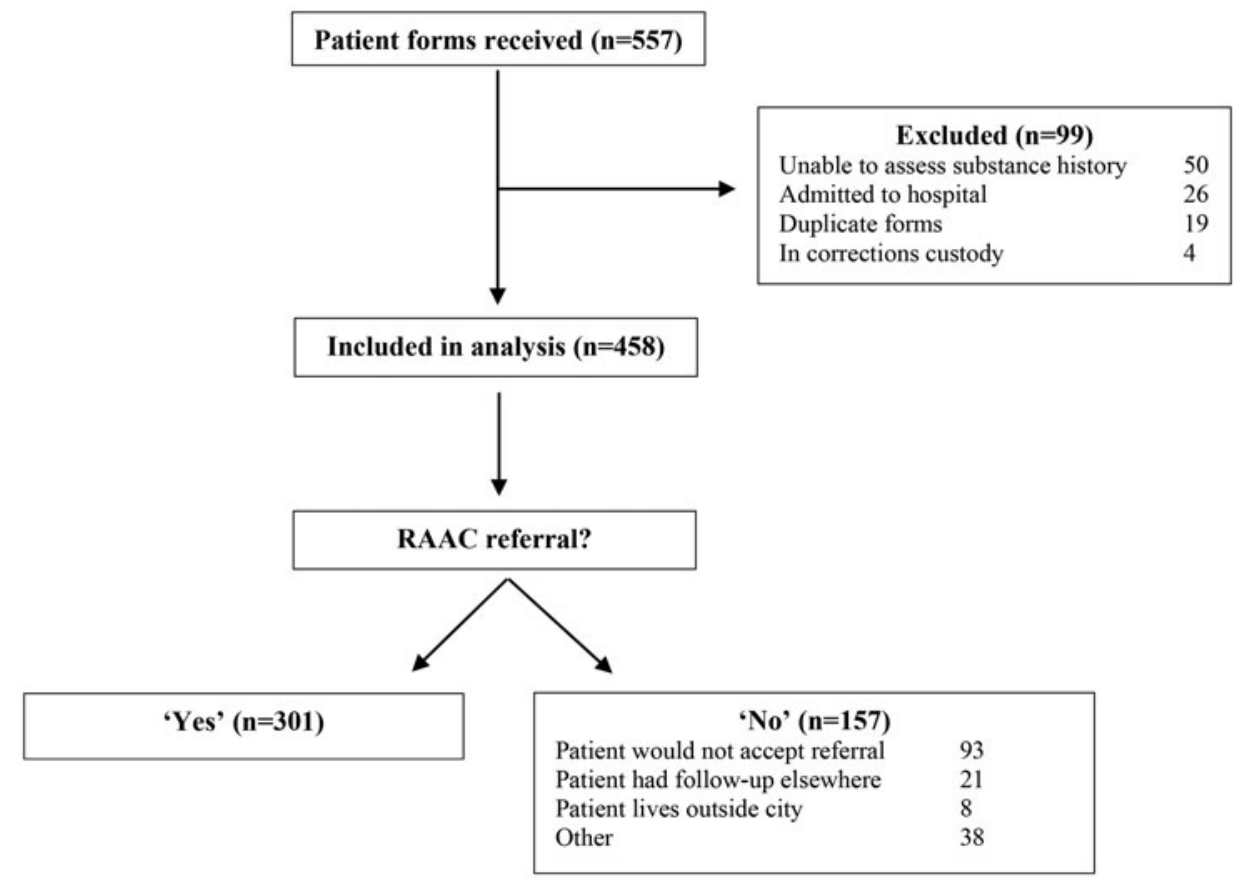

Figure 1. Patient flow. $\mathrm{RAAC}=$ Rapid-access addiction clinic.

several themes, such as lack of patient insight into their problematic substance use, being unsure if the patient's substance use could be classified as problematic or highrisk, or the patient's substance use was an isolated event (no evidence of chronic substance use).

Descriptive statistics for patient demographics and ED visit characteristics are displayed in Table 1. Median age for enrolled patients was 35 years (IQR, 28-42 years), and $64.4 \%$ were male. Median age for all patients discharged from the ED during the study period was 49 years (IQR, 32-66 years), and 50.9\% were male. Alcohol was the most common substance indicated for enrolled patients $(60.2 \%)$, followed by stimulants $(31.9 \%)$, and opioids $(15.9 \%)$. Polysubstance use was identified in $22.7 \%$ of enrolled patients. The median ED length of stay of enrolled patients was 8 hours 3 minutes (IQR, 5 hours 34 minutes - 11 hours 7 minutes), compared with 5 hours 54 minutes (IQR, 3 hours 41 minutes -8 hours 45 minutes) for all-comers discharged from the ED during the study period. Over one-quarter of enrolled patients had made a previous ED visit within 7 days before their index visit $(\mathrm{n}=127 ; 27.7 \%)$, with an average of 1.8 additional visits per patient $(\mathrm{SD}=1.2)$. Comparatively, $18.4 \%$ of all-comers discharged from the ED during the study period made a previous visit within 7 days. An additional $16.8 \%$ of enrolled patients had a previous ED visit within 30 days before their index visit.

We examined the full multivariable logistic regression model and compared performance of c-index and accompanying regression diagnostics with the parsimonious and final model comprised of age, length of stay, triage complaint category, day of week of triage, discharge time, and substance of use. Table 2 displays aORs with corresponding $95 \%$ CIs for the main effects of the final model with a c-index of 0.7 that predicted patient referral to a hypothetical rapid-access addiction clinic from the ED. Patient sex, CTAS score, triage time, and repeat ED visits in the previous 7 days and 30 days did not improve the model fit nor were their respective coefficients significant; thus, these variables were removed from the final parsimonious model. Triage time, length of stay, and discharge time are deterministic and, therefore, exhibit multicollinearity if all three are included. Stepwise forward selection revealed that length of stay and discharge time provided the best fit for the final model. Substance-related triage complaint categories were associated with higher likelihood of referral $(\mathrm{OR}=2.00 ; 95 \% \mathrm{CI}, 1.21-3.30)$. Patients indicated as using opioids only, stimulants only, "other substance" only, and polysubstance use all had an increased likelihood of rapid-access addiction clinic 


\begin{tabular}{|c|c|c|}
\hline Characteristics & $N=458$ & $\%=100$ \\
\hline Age, median (IQR), years & \multicolumn{2}{|c|}{35.0 (28 to 47 ) } \\
\hline \multicolumn{3}{|l|}{ Sex } \\
\hline Female & 163 & 35.6 \\
\hline Male & 295 & 64.4 \\
\hline \multicolumn{3}{|l|}{ CTAS $^{b}$} \\
\hline 1 & 8 & 1.7 \\
\hline 2 & 162 & 35.4 \\
\hline 3 & 207 & 45.2 \\
\hline 4 & 70 & 15.3 \\
\hline 5 & 11 & 2.4 \\
\hline \multicolumn{3}{|l|}{ Triage complaint category } \\
\hline Substance-related & 136 & 29.7 \\
\hline Overdose ingestion & 26 & 21.2 \\
\hline Substance misuse/intoxication & 85 & 49.1 \\
\hline Substance withdrawal & 25 & \\
\hline Mental health-related ${ }^{c}$ & 97 & \\
\hline Other & 225 & \\
\hline All trauma & 44 & \\
\hline Major trauma (blunt) & 27 & \\
\hline \multicolumn{3}{|l|}{ Substance indicated } \\
\hline \multicolumn{3}{|l|}{ Alcohol } \\
\hline Alcohol only & 202 & 44.1 \\
\hline Alcohol total & 276 & 60.2 \\
\hline \multicolumn{3}{|l|}{ Cannabis } \\
\hline Cannabis only & 10 & 2.2 \\
\hline Cannabis total & 38 & 8.3 \\
\hline \multicolumn{3}{|l|}{ Opioids } \\
\hline Opioids only & 41 & 8.9 \\
\hline Opioids total & 73 & 15.9 \\
\hline \multicolumn{3}{|l|}{ Stimulants } \\
\hline Stimulants only & 74 & 16.2 \\
\hline Stimulants total & 146 & 31.9 \\
\hline \multicolumn{3}{|l|}{ Other ${ }^{d}$} \\
\hline Other only & 12 & 2.6 \\
\hline Other total & 42 & 9.2 \\
\hline Polysubstance $e^{\mathrm{e}}$ & 104 & 22.7 \\
\hline None specified & 15 & 3.3 \\
\hline \multicolumn{3}{|l|}{ Day of week } \\
\hline Monday & 63 & 13.8 \\
\hline Tuesday & 61 & 13.3 \\
\hline Wednesday & 58 & 12.7 \\
\hline Thursday & 80 & 17.5 \\
\hline Friday & 54 & 11.8 \\
\hline Saturday & 82 & 17.9 \\
\hline Sunday & 60 & 13.1 \\
\hline \multicolumn{3}{|l|}{ Triage time ${ }^{f}$} \\
\hline Morning & 83 & 18.1 \\
\hline Afternoon & 109 & 23.8 \\
\hline Evening & 140 & $\begin{array}{c}30.6 \\
\text { ontinued) }\end{array}$ \\
\hline
\end{tabular}

\begin{tabular}{|c|c|c|}
\hline Characteristics & $N=458$ & $\%=100$ \\
\hline Overnight & 126 & 27.5 \\
\hline Length of stay, median (IOR), minutes & \multicolumn{2}{|c|}{483 (334 to 667) } \\
\hline \multicolumn{3}{|l|}{ Discharge time $e^{f}$} \\
\hline Morning & 151 & 33.0 \\
\hline Afternoon & 139 & 30.3 \\
\hline Night & 88 & 19.2 \\
\hline Overnight & 80 & 17.5 \\
\hline \multicolumn{3}{|l|}{ Repeat ED visits } \\
\hline Previous visit within 7 days only & 127 & 27.7 \\
\hline Previous visit within 30 days only & 77 & 16.8 \\
\hline Previous visit within 7 days \& 30 days & 13 & 2.9 \\
\hline None & 241 & 52.6 \\
\hline \multicolumn{3}{|c|}{$\begin{array}{l}\text { "Demographic and ED visit characteristics of patients identified as having problematic or } \\
\text { high-risk substance use who visited the University of Alberta ED between June } 13^{\text {th }} \text { and } \\
\text { August } 21^{\text {st }} 2018 \text {. } \\
\text { bThe CTAS is a scale from } 1 \text { to } 5 \text {, whereby } 1 \text { is the highest level of acuity and } 5 \text { is the lowest } \\
\text { level of acuity. } \\
\text { "Mental health-related: "anxiety/situational crisis," "bizarre behavior," "depression/ } \\
\text { suicide/deliberate self-harm," "hallucinations/delusions."' } \\
\text { dOther substance indicated: benzodiazepines, nicotine, anabolic steroids, } \\
\text { gamma-hydroxybutyrate (GHB), dimenhydrinate, hallucinogens, methocarbamol, 'paint } \\
\text { thinner', sedative/hypnotic, butane hash oil (BHO; "shatter"). } \\
\text { 'Polysubstance: greater than one substance indicated. } \\
\text { 'Triage time and Discharge time: morning 06:00-11:59, afternoon 12:00-17:59, evening } \\
\text { 18:00-23:59, overnight 00:00-05:59. }\end{array}$} \\
\hline
\end{tabular}

referral from the ED. Age, length of stay, day of week, and discharge time did not have a statistically significant effect on likelihood of patient referral.

\section{DISCUSSION}

\section{Interpretation of findings}

We found that substance-related ED presentations were common, with a mean of 4.3 patients per day identified for referral to a hypothetical rapid-access addiction clinic during the study period. This indicates a gap in care that warrants attention. ED patients identified as using any substance (except cannabis) and patients who presented with a substance-related triage complaint category were more likely to be referred. Approximately half of included patients had a triage complaint category that was not directly substance-related. This demonstrates the pervasive nature of substance use among ED patients and how it may be a primary or secondary contributing factor to any ED visit.

As noted in previous studies, patients using substances were more often young and male. ${ }^{1,20,23,24}$ Alcohol was the most common substance indicated, a finding which 


\begin{tabular}{|c|c|c|}
\hline & \multicolumn{2}{|c|}{ Logistic regression } \\
\hline & aOR $(95 \% \mathrm{CI})^{\mathrm{b}}$ & $p$-Value \\
\hline Triage complaint category & & 0.01 \\
\hline Other (reference) & 1 & \\
\hline Substance-related ${ }^{c}$ & $2.0(1.2,3.3)$ & \\
\hline Mental health-related ${ }^{d}$ & $1.7(0.99,3.1)$ & \\
\hline Substance indicated & & 0.002 \\
\hline Alcohol only (reference) & 1 & \\
\hline Cannabis only & $0.1(0.0,0.7)$ & \\
\hline Opioids only & $1.5(0.7,3.4)$ & \\
\hline Stimulants only & $1.2(0.6,2.2)$ & \\
\hline Other only ${ }^{\mathrm{e}}$ & $2.8(0.6,13.3)$ & \\
\hline Polysubstance $^{f}$ & $1.3(0.8,2.3)$ & \\
\hline None & $0.1(0.0,0.5)$ & \\
\hline \multicolumn{3}{|c|}{$\begin{array}{l}\text { aLikelihood of ED patient referral to an on-site rapid-access addiction clinic from the } \\
\text { University of Alberta Hospital emergency department (ED) from June } 13^{\text {th }} \text { to August } 21^{\text {st }} \\
2018 \text {. } \\
\text { 'The aORs with 95\% Cls: model is adjusted for patient age in years, discharge time } \\
\text { (morning, afternoon, night, overnight), ED length of stay, and day of week of discharge. } \\
\text { "Substance-related: "overdose ingestion." "substance misuse/intoxication," "substance } \\
\text { withdrawal." } \\
\text { dMental health-related: "anxiety/situational crisis," "bizarre behavior," "depression/ } \\
\text { suicide/deliberate self-harm," "hallucinations/delusions." } \\
\text { eOther substance indicated: benzodiazepines, nicotine, anabolic steroids, } \\
\text { gamma-hydroxybutyrate (GHB), dimenhydrinate, hallucinogens, methocarbamol, "paint } \\
\text { thinner," sedative/hyphotic, butane hash oil (BHO; "shatter"). } \\
\text { 'Polysubstance: greater than one substance indicated. }\end{array}$} \\
\hline
\end{tabular}

is consistent with previous literature..$^{20,25,26}$ The study hospital is not the primary hospital of presentation for patients using opioids in this urban center, given that it is located outside the downtown inner-city area, where substance use is more heavily concentrated and the rate of opioid overdose is highest. ${ }^{1,2}$ Despite this, opioids were identified in almost $16 \%$ of our study cohort. Provincial data from 2017 indicated that $17 \%$ of patients who died from accidental opioid poisoning related to fentanyl made an ED visit related to substance use in the 30 days preceding their death. ${ }^{1}$ This further supports the role of the ED in identifying and intervening upon patients at risk of morbidity and mortality consequences related to substance use.

Patients with substance-related ED presentations spent longer in the ED than all-comers who were discharged from the ED during the study period (8 hours 3 minutes v. 5 hours 54 minutes). A study from Vancouver, Canada (2003), also found that patients presenting with substance-related problems had a longer median ED length of stay ( 3 hours 52 minutes v. 2 hours 44 minutes). ${ }^{10}$

Many previous studies have shown that patients who use substances use ED services at higher frequency compared with the general population., ${ }^{9,24,27}$ In this study, over one-quarter of patients made a previous ED visit in the 7 days before their index visit. Linking these patients to an on-site addiction clinic on the same day of their ED visit could be beneficial to both the patient and the hospital system. Several North American studies have shown that connecting patients from hospital to specialized addiction resources leads to a reduction in repeat ED visits. ${ }^{16-21}$

\section{STRENGTHS AND LIMITATIONS}

A strength of this study is its timely effort to address a public health crisis and assess need for increasing services to a currently underserved population. It provides an important update on current substance use patterns in the ED. The study also has a pragmatic protocol and could be easily replicated at other sites.

We acknowledge some limitations to the study. First, it was a single-center study, limiting the generalizability of the results. It was also a chart review, introducing potential for inaccuracies in chart interpretation, which would reduce internal validity. There was only one reviewer abstracting chart data, so it was not possible to assess inter-rater agreement. Ongoing prospective evaluation of this cohort would have been preferable for detecting subsequent ED presentations and morbidity and mortality outcomes. Unfortunately, ethics approval for an ongoing prospective study was incompatible with the privacy laws of the provincial electronic medical record.

Additionally, a formal screening process for substance use disorders was not used, meaning that some eligible patients may have been missed for enrolment. The decision not to use formal screening was made to be pragmatic and cognizant of time restraints on the already numerous responsibilities of the ED care team, and to reflect existing clinical circumstances at this ED in which formal screening for substance use is not currently used. Furthermore, given that the clinic is only "hypothetical" and not an actual clinic, some health care providers may have been less motivated to enroll patients into the study, given that there would be no tangible outcome change for their patient at that ED visit. Taking all of this into account, the results are likely an underestimation of the true volume of substance-related ED presentations at this hospital. Lastly, the patients in the study were not specifically asked if they would accept referral 
to a hypothetical rapid-access addiction clinic, which reduces the practical applicability of the results. While it is certainly important to include patients in decisionmaking and resource development, collecting additional data from patients would have required a stricter ethics consent policy for the study, which was decided against to remove barriers to patient enrolment.

\section{CONCLUSION}

This prospectively enrolled cohort study characterized substance-related ED presentations at an urban Canadian tertiary care trauma center. At least four patients per day would have been referred to an on-site rapid-access addiction clinic, had one been available. Considering that this is likely an underestimation of the true volume of patients who would be eligible for referral, this study draws attention to a growing public health issue. Development of a rapid-access addiction clinic for this patient population is currently being explored. This study provides a model for other centers to modify and replicate to identify and address specific unmet needs of their respective patient populations.

Supplementary material: The supplementary material for this article can be found at https://doi.org/10.1017/cem.2019.453.

Acknowledgements: Thank you to the Alberta Health Services Emergency Strategic Clinical Network and Mr. Anton Van Niekerk of Alberta Health Services for guidance in the statistical analysis for this study.

Financial support: Funding for Mr. Howie $\mathrm{Wu}$ was obtained through an Alberta Health Services Emergency Strategic Clinical Network Undergraduate Summer Studentship Award 2018.

Competing interests: None declared.

\section{REFERENCES}

1. Alberta Government [Internet]. Opioid and substances of misuse: Alberta Report 2017 Q4; March 2, 2018; [about 28 screens]. Available at: https://open.alberta.ca/publications/ opioids-and-substances-of-misuse-alberta-report (accessed October 31, 2019).

2. Alberta Government [Internet]. Opioid and substances of misuse: Alberta Report 2018 Q4; March 2019; [about 37 screens]. Available at: https://open.alberta.ca/publications/ opioids-and-substances-of-misuse-alberta-report (accessed October 31, 2019).

3. Government of Canada [Internet]. Overview of national data on opioid-related harms and deaths; December 12, 2018; [about 1 screen]. Available at: https://www.canada.ca/en/ health-canada/services/substance-use/problemativ-prescription- drug-use/opioids/data-surveillance-research/harms-deaths. html (accessed October 31, 2019).

4. Canadian Institute for Health Information [Internet]. Hospitalizations and emergency department visits due to opioid poisoning in Canada; 2016; [about 31 screens]. Available at: https://secure.cihi.ca/free_products/Opioid\%20Poisoning\% 20Report\%20\%20EN.pdf (accessed October 31, 2019).

5. Young L. Opioids death toll in Canada nearly 4,000 last year, new data show. Canadian Broadcasting Company [Internet]. June 19, 2018; Life: [about 3 screens]. Available at: https:// www.cbc.ca/news/politics/opioid-death-toll-marketing-1. 4712419 (accessed October 31, 2019).

6. National Safety Council [Internet]. Injury facts: all injuries; 2017; [about 2 screens]. Available at: https://injuryfacts.nsc. org/all-injuries/overview (accessed October 31, 2019).

7. Fowler K, Senthilingam M. Odds of dying from accidental opioid overdose in the US surpass those of dying in car accident. Cable News Network [Internet]. January 14, 2019; Life: [about 1 screen]. Available at: https://www.cnn.com/ 2019/01/14/health/opioid-deaths-united-states-surpass-roadaccidents/index.html (accessed October 31, 2019).

8. Centre for Disease Control [Internet]. CDC's efforts to prevent opioid overdoses and other opioid-related harms; January 2019; [about 2 screens]. Available at: https://www. cdc.gov/opioids/pdf/Strategic-Framework-Factsheet_Jan2019_ 508.pdf (accessed October 31, 2019).

9. Kendall CE, Boucher LM, Mark AE, et al. A cohort study examining emergency department visits and hospital admissions among people who use drugs in Ottawa, Canada. Harm Reduction 7 2017;14(1):16.

10. Brubacher JR, Mabie A, Ngo M, et al. Substance-related problems in patients visiting an urban Canadian emergency department. CFEM 2008;10(3):198-204.

11. Martin A, Mitchell A, Wakeman S, White B, Raja A. Emergency department treatment of opioid addiction: an opportunity to lead. Acad Emerg Med 2018;25(5):601-4.

12. Morris DW, Ghose S, Williams E, Brown K, Khan F. Evaluating psychiatric readmissions in the emergency department of a large public hospital. Neuropsychiatr Dis Treat 2018;14:671-9.

13. Elliott K, W Klein J, Basu A, Sabbatini AK. Transitional care clinics for follow-up and primary care linkage for patients discharged from the ED. Am $\mathcal{F}$ Emerg Med 2016; 34(7):1230-5.

14. Breton AR, Taira DA, Burns E, O'Leary J, Chung RS. Follow-up services after an emergency department visit for substance abuse. Am 7 Manag Care 2007;13(9):497-505.

15. Weiner SG, Baker O, Bemson D, Schuur JD. One-year mortality of opioid overdose victims who received naloxone by emergency medical services. Ann Emerg Med 2017;70(4S) (Abstract).

16. Andrews CM, Westlake M, Wooten N. Availability of outpatient addiction treatment and use of emergency department services among Medicaid enrollees. Psychiatr Serv 2018;69(6):729-32.

17. Pecoraro A, Horton T, Ewen E, et al. Early data from Project Engage: a program to identify and transition medically hospitalized patients into addictions treatment. Addict Sci Clin Pract 2012;7:20. 
18. O’Toole TP, Pollini RA, Ford DE, Bigelow G. The effect of integrated medical-substance abuse treatment during an acute illness on subsequent health services utilization. Med Care 2007;45(11):1110-5.

19. Friedmann PD, Hendrickson JC, Gerstein DR, Zhang Z, Stein MD. Do mechanisms that link addiction treatment patients to primary care influence subsequent utilization of emergency and hospital care? Med Care 2006;44(1):8-15.

20. Palepu A, Horton NJ, Tibbetts N, Dukes K, Meli S, Samet JH. Substance abuse treatment and emergency department utilization among a cohort of $\mathrm{HIV}$-infected persons with alcohol problems. F Subst Abuse Treat 2003;25(1):37-42.

21. Englander H, Weimer M, Solotaroff R, et al. Planning and designing the Improving Addiction Care Team (IMPACT) for hospitalized adults with substance use disorder. 7 Hosp Med 2017;12(5):339-42.

22. Beveridge R, Clarke B, Janes L, et al. Canadian Emergency Department Triage and Acuity Scale: implementation guidelines. Can 7 Emerg Med 1999;1(3 Suppl):S2-S28.

23. Otterstatter MC, Crabtree A, Dobrer S, et al. Patterns of health care utilization among people who overdosed from illegal drugs: a descriptive analysis using the BC Provincial Overdose Cohort. Health Promot Chronic Dis Prev Can 2018;38(9):328-33.

24 Urbanoski K, Cheng J, Rehm J, Kurdyak P. Frequent use of emergency departments for mental and substance use disorders. Emerg Med $\mathcal{Z}$ 2018;35(4):220-5.

25. Howard R, Fry S, Chan A, Ryan B, Bonomo Y. A feasible model for early intervention for high-risk substance use in the emergency department setting. Aust Health Rev 2019; 43(2):188-93.

26. Blow FC, Walton MA, Barry KL, et al. Alcohol and drug use among patients presenting to an inner-city emergency department: a latent class analysis. Addict Bebav 2011;36(8): 793-800.

27. Zhang L, Norena M, Gadermann A, et al. Concurrent disorders and health care utilization among homeless and vulnerably housed persons in Canada. 7 Dual Diagn 2018;14(1):21-31.

28. Hensel JM, Taylor VH, Fung K, Yang R, Vigod SN. Acute care use for ambulatory care-sensitive conditions in high-cost users of medical care with mental illness and addictions. Can 7 Psychiatry 2018, doi: 10.1177/0706743717752880. 